\title{
Anomalous periodicity of magnetic interference patterns in encapsulated graphene Josephson junctions
}

\author{
C. T. Ke,${ }^{1,{ }^{*}}$ A. W. Draelos,,${ }^{1,}$ A. Seredinski ${ }^{1,}{ }^{*}$ M. T. Wei,${ }^{1}$ H. Li,${ }^{2}$ M. Hernandez-Rivera, ${ }^{2}$ K. Watanabe,${ }^{3}$ T. Taniguchi, ${ }^{3}$ \\ M. Yamamoto, ${ }^{4}$ S. Tarucha ${ }^{4}$ Y. Bomze, ${ }^{1}$ I. V. Borzenets $\odot,{ }^{5, \dagger}$ F. Amet,${ }^{2}$ and G. Finkelstein ${ }^{1}$ \\ ${ }^{1}$ Department of Physics, Duke University, Durham, North Carolina 27708, USA \\ ${ }^{2}$ Department of Physics and Astronomy, Appalachian State University, Boone, North Carolina 28607, USA \\ ${ }^{3}$ Advanced Materials Laboratory, National Institute for Materials Science, 1-1 Namiki, Tsukuba 305-0044, Japan \\ ${ }^{4}$ Center for Emergent Matter Science (CEMS), RIKEN, Wako-shi, Saitama 351-0198, Japan \\ ${ }^{5}$ Department of Physics, City University of Hong Kong, Kowloon, Hong Kong SAR
}

(Received 19 June 2019; revised manuscript received 30 September 2019; published 7 November 2019)

\begin{abstract}
We investigate supercurrent interference patterns measured as a function of magnetic field in ballistic graphene Josephson junctions. At high doping, the expected $\Phi_{0}$-periodic "Fraunhofer" pattern is observed, indicating a uniform current distribution. Close to the Dirac point, we find anomalous interferences that are close to a $2 \Phi_{0}$ periodicity, similar to that predicted for topological Andreev bound states carrying a charge of $e$ instead of $2 e$. This feature persists with increasing temperature, ruling out a nonsinusoidal current-phase relationship. It also persists in junctions in which sharp vacuum edges are eliminated. Our results indicate that the observed behavior may originate from an intrinsic property of ballistic graphene Josephson junctions, though the exact mechanism remains unclear.
\end{abstract}

DOI: 10.1103/PhysRevResearch.1.033084

The critical current of a Josephson junction subject to a perpendicular magnetic field is known to show decaying oscillations [1,2]. For a uniform supercurrent distribution and a $2 \pi$-periodic sinusoidal current-phase relation, the pattern of oscillations is identical to that of single-slit Fraunhofer interference in optics. This pattern's periodicity is $\Phi_{0}=h / 2 e$, the magnetic flux quantum. Measurement of (and deviations from) the "Fraunhofer pattern" is a conventional way to characterize the uniformity of Josephson junctions which became particularly relevant for the novel junctions based on $2 \mathrm{D}$ materials [3-6].

Josephson junctions made with topological materials, such as quantum spin Hall insulators, demonstrate marked deviations from the conventional Fraunhofer pattern [7]. Topological bound states at the superconducting interface are able to support the supercurrent along the edges of the sample, resulting in SQUID-like oscillations. Due to the presence of Majorana fermions, these patterns are expected to show a single electron periodicity of $h / e$ [8-10]. However, due to quasiparticle poisoning, this periodicity may not be typically accessible via DC measurements [11-13]. Nevertheless, magnetic interference patterns with even-odd modulations have been reported [14-16]. Other scenarios are also expected

\footnotetext{
*These authors contributed equally to this work.

†iborzene@cityu.edu.hk
}

Published by the American Physical Society under the terms of the Creative Commons Attribution 4.0 International license. Further distribution of this work must maintain attribution to the author(s) and the published article's title, journal citation, and DOI. to result in distortions of the interference pattern, including the presence of a nonuniform supercurrent distribution [17-20], a nonsinusoidal current-phase relation [21-24], spinorbit effects [25-27], and a nonlocal supercurrent [28-34]. Understanding these transport mechanisms may allow one to distinguish trivial $2 \Phi_{0}$-periodic behavior from topological $2 \Phi_{0}$-periodic behavior.

Here, we study encapsulated graphene Josephson junctions in several different regimes. We start by measuring the samples at high density, where we find conventional $\Phi_{0}$-periodic Fraunhofer patterns. As the density is lowered, the junctions exhibit a robust lifting of even nodes, resulting in effectively $2 \Phi_{0}$-periodic interference patterns. The patterns with even node lifting were repeatedly observed in several devices of different geometries, making it highly unlikely that the behavior is a result of an aberrant, nonuniform current density. Furthermore, the observed behavior is unaffected by side gates that change the density near the junction/vacuum edges. The anomalous patterns persist at elevated temperature where the current-phase relation is expected to be sinusoidal $[23,24]$. Note that this system is not expected to host any topological bound states due to its lack of spin-orbit coupling. Having ruled out the above scenarios, we suggest that this anomalous periodicity may originate from some intrinsic properties of ballistic graphene Josephson junctions.

We study seven Josephson junctions made of graphene encapsulated in hexagonal boron nitride and contacted by molybdenum-rhenium (MoRe) superconducting electrodes. The junction dimensions are listed in Table I, while fabrication and measurement details are included in the appendices. The junctions have different lengths, $L$, and contact widths, $W$. Junctions $J_{1-5}$ are conventional rectangular junctions, while 
TABLE I. List of junctions.

\begin{tabular}{lccc}
\hline \hline Device name & Length & Width & Ratio: $L / W$ \\
\hline$J_{1}$ & $0.65 \mu \mathrm{m}$ & $4.5 \mu \mathrm{m}$ & 0.144 \\
$J_{2}$ & $0.3 \mu \mathrm{m}$ & $2.4 \mu \mathrm{m}$ & 0.125 \\
$J_{3}$ & $0.2 \mu \mathrm{m}$ & $3 \mu \mathrm{m}$ & 0.067 \\
$J_{4}$ & $0.4 \mu \mathrm{m}$ & $3 \mu \mathrm{m}$ & 0.133 \\
$J_{5}$ & $1 \mu \mathrm{m}$ & $3 \mu \mathrm{m}$ & 0.333 \\
$J_{\text {side }}$ & $0.5 \mu \mathrm{m}$ & $3 \mu \mathrm{m}$ & 0.166 \\
$J_{\text {ex }}$ & $0.5 \mu \mathrm{m}$ & $3 \mu \mathrm{m}$ & 0.166 \\
\hline \hline
\end{tabular}

$J_{\text {side }}$ and $J_{\mathrm{ex}}$ include local electrostatic gates along the edges of the junctions. The ballisticity of junctions $J_{1-5}$ was established in depth in a previous study [35]. For the first part of this paper we focus on $J_{1}$ for clarity and brevity.

The differential resistance of $J_{1}$ as a function of applied DC bias current $I$ and back gate voltage $V_{\mathrm{G}}$ is shown in Fig. 1(a). The black region roughly symmetric about $I=0$ corresponds to the superconducting state, in which the junction resistance vanishes. The transition from the superconducting state to the normal state occurs at the switching current, $I_{\mathrm{S}}$. At high carrier density, the switching current increases proportionally with the number of conducting modes; it reduces to its minimum around the Dirac point $\left(V_{\mathrm{D}} \approx-2.65 \mathrm{~V}\right)$. Conventional Fraunhofer patterns are observed at high electron density in all junctions [Fig. 1(b)]. In $J_{1}$, this regime persists for densities $n \gtrsim 5 \times 10^{10} \mathrm{~cm}^{-2}\left(V_{\mathrm{G}}-V_{\mathrm{D}} \gtrsim 0.7 \mathrm{~V}\right)$. This is shown in Fig. 1(c), which demonstrates that the oscillations remain unchanged for most of the $V_{\mathrm{G}}$ range. Due to the possibility of flux trapping in leads which would distort the pattern and introduce hysteresis [36], the magnetic field is kept within the $\pm 5 \mathrm{mT}$ range for these and other measurements. In the case of hole doping, $p n$ junctions are formed in the graphene due to local $n$ doping by the MoRe contacts. This results in Fabry-Pérot oscillations, which manifest as resonances in $I_{\mathrm{S}}$ measured versus $V_{\mathrm{G}}[32,37]$ (see Appendix B). In this regime, anomalies in the Fraunhofer pattern are clear, as seen in the contrast between Figs. 1(c) and 1(d). Previous

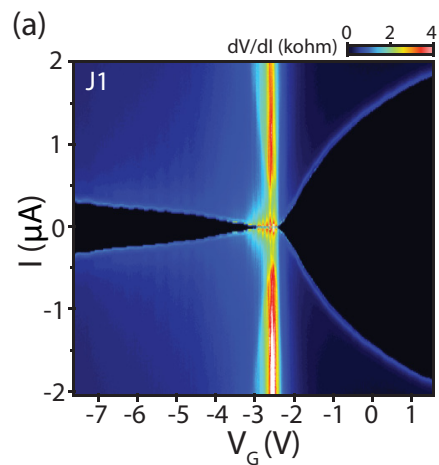

(b)
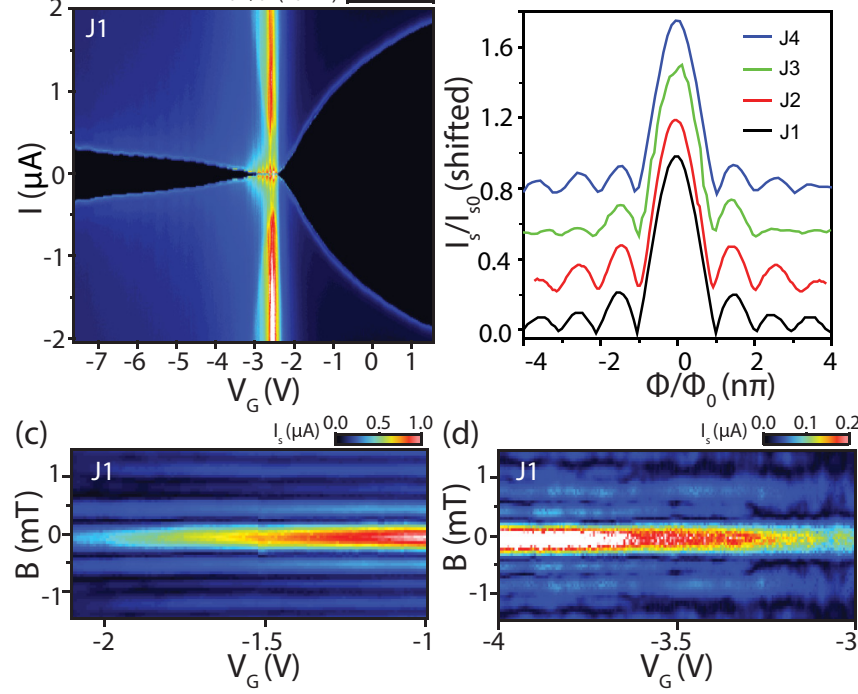

FIG. 1. Conventional graphene Josephson junction behavior. (a) Map of differential resistance $d V / d I$ of junction $J_{1}$ as a function of applied current $I$ and back gate voltage $V_{\mathrm{G}}$. The dark region of vanishing resistance near zero bias corresponds to the supercurrent. (b) Regular Fraunhofer interference patterns measured at high density for $J_{1-4}$. (c) Maps of supercurrent $I_{\mathrm{S}}$ in $J_{1}$ versus magnetic field $B$ and gate voltage $V_{\mathrm{G}}$, taken at high electron doping. These interference maps demonstrate conventional Fraunhofer patterns with a gateindependent magnetic oscillation period. (d) A similar map in the hole-doped regime demonstrating deviations from the Fraunhofer pattern closer to the Dirac point $\left(V_{\mathrm{D}}=-2.65 \mathrm{~V}\right)$ and the restored Fraunhofer pattern farther away.

work has attributed these anomalous patterns to different Fabry-Pérot resonances in bulk and edge modes, resulting in edge-dominated (SQUID-like) interference when the bulk transmission is low [18].

In this paper, we explore the regime of very small densities, $n \lesssim 2.5 \times 10^{10} \mathrm{~cm}^{-2}$, presented in Fig. 2(a). Here, we find that $2 \Phi_{0}$-periodic interference patterns arise as shown in
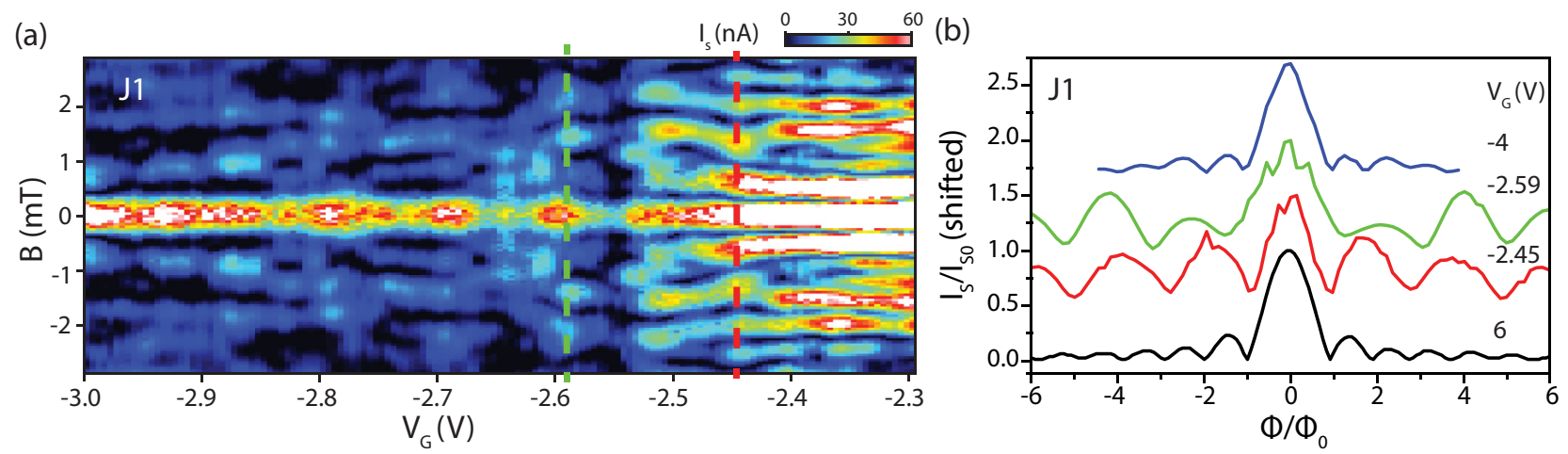

FIG. 2. Interference patterns with periodicity doubling. (a) Magnetic interference measurements at low doping for $J_{1}$. Oscillations in the switching current along the gate direction for $p$ doping result from Fabry-Pérot resonances in the junction due to $p n$ interfaces near the MoRe contacts. Green and red lines mark cuts displayed in adjacent panel. (b) Line cuts showing normalized $I_{\mathrm{S}}$ as a function of quantized magnetic flux $\Phi / \Phi_{0}$. At high electron (black) and hole (blue) doping, a regular oscillation period is observed. However, for certain $V_{\mathrm{G}}$ near the Dirac point (red, green) we find a regular pattern of oscillations with a doubled periodicity. 
Fig. 2(b), which correspond to the vertical cross sections of Fig. 2(a) at $V_{\mathrm{G}}=-2.45 \mathrm{~V}$ and $-2.59 \mathrm{~V}$. In these two curves (red and green), all even nodes are completely lifted and the overall periodicity changes from $0.35 \mathrm{mT}$ to $0.68 \mathrm{mT}$ as compared to results at high electron and hole density (blue and black). The interference patterns retain this regular $2 \Phi_{0}$ period for several flux quanta and thus the pattern change cannot be explained by a randomly distorted current distribution. The regions of periodicity doubling also persist for a significant range of $V_{\mathrm{G}}$ [Fig. 2(a)], further indicating that disordered current distribution is not likely to be the cause.

We would like to point out that as a result of the pattern change, the width of the side lobes becomes roughly equal to the width of the central peak, whose width stays constant. While a central maximum with the same width as the side maxima indicates a SQUID-like interference pattern, the observed behavior would correspond to a SQUID with a $4 \pi$-periodic current-phase relation (CPR). However, previous CPR studies on ballistic graphene Josephson junctions show a $2 \pi$-periodic CPR persisting through the Dirac point $[23,24]$. Alternatively, $2 \Phi_{0}$-periodic interference pattern could be the result of crossed Andreev reflections, in which the electron and the Andreev-reflected hole propagate along the opposite sides of the junction, as discussed in Ref. [20] for topologically trivial InAs. We explore (and refute) possible contributions from the edges in our samples in the following.

Previous studies have demonstrated a significant density buildup along the vacuum edges of graphene devices [38-40]. In order to determine whether edge effects are responsible for the anomalous interference patterns, we employ two types of local gates which directly affect the edge carrier density. In $J_{\text {side }}$, self-aligned side gates are made from the same graphene crystal as the Josephson junction by etching a narrow gap $(\lesssim 100 \mathrm{~nm})$ between the two [Fig. 3(a)] [41]. As the side gates are very close to the edge of the junction, they are highly efficient, allowing us to change the local density by approximately $\pm 4 \times 10^{11} \mathrm{~cm}^{-2}$ relative to the bulk. In $J_{\mathrm{ex}}$, the graphene mesa extends several microns beyond the junction on both edges. The carrier densities of these extended regions are controlled by two local top gates, which come to within $\sim 100 \mathrm{~nm}$ of the contacts and tune the local density by $\pm 5 \times$ $10^{12} \mathrm{~cm}^{-2}$ [Fig. 3(b)]. The length of the junction edge is much greater than the induced superconducting coherence length in graphene, $L_{\text {edge }} \gg \xi_{0}=\hbar v_{F} / \Delta \sim 500 \mathrm{~nm}$. In this regime the critical current at the lowest temperatures is expected to scale as $I_{C} \propto 1 / L[35,42-44]$, and the contribution of the edges to supercurrent is expected to be negligible in this sample.

Both $J_{\text {side }}$ and $J_{\text {ex }}$ show lifting of even nodes near the bulk Dirac point, while the first and third nodes remain pronounced. Interestingly, the first node also remains pinned at a fixed value of $\Phi=\Phi_{0}$ while the rest of the pattern is strongly distorted. This observed anomalous periodicity is robust and persists over the full range of side or top gate voltages. In fact, the interference maps as a function of gate voltage and magnetic field [Figs. 3(c) and 3(d)] remain roughly unchanged as the top or side gates are applied, except for a small shift along the back gate axis, induced by their overall electrostatic influence.
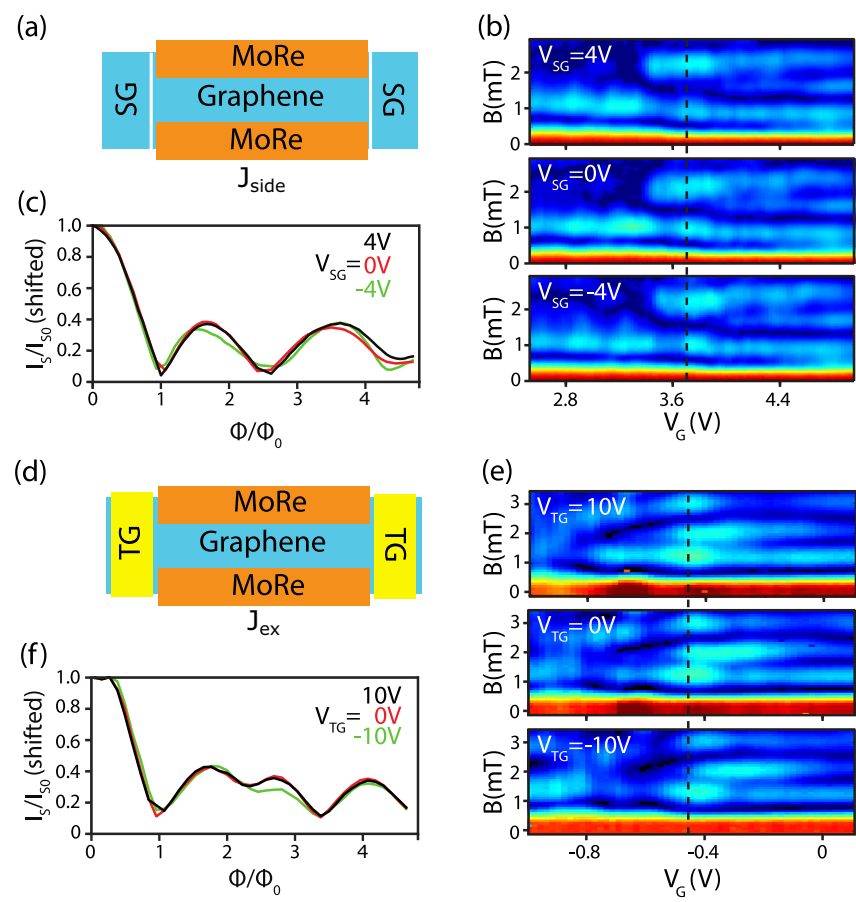

FIG. 3. Interference in junctions with edge density control. (a) Schematic of $J_{\text {side }}$ showing two side gates which modify the potential at the vacuum edges. (b) The normalized supercurrent as a function of magnetic field and gate voltages in $J_{\text {side }}$. The density on both edges is increased (decreased) by $\sim 4 \times 10^{11} \mathrm{~cm}^{-2}$ for $V_{\mathrm{SG}}=4 \mathrm{~V}(-4 \mathrm{~V})$. Little influence on the interference pattern is seen. (c) Line cuts from the dashed lines in (b) showing lifting of the even nodes. (d) Schematic of $J_{\mathrm{ex}}$ with extended areas on both sides of the mesa and top gates to control the local density. (e) As (b) but for $J_{\mathrm{ex}}$, applying identical voltages to both local top gates. Here, the local density is tuned dramatically by $\sim 5 \times 10^{12} \mathrm{~cm}^{-2}$ for $V_{\mathrm{TG}}= \pm 10 \mathrm{~V}$. Again, the influence on the interference pattern is minor. (f) Line cuts from the dashed line in (e). Again, there is no influence on the anomalous period by the local gates. Hence we conclude that the anomalous periodicity is not caused by trivial edge channels.

In particular, the anomalous pattern persists in $J_{\text {side }}$ [Figs. 3(c) and 3(e)] as the side gate voltages are applied in both the positive and negative directions, such that the edges of the junction acquire a carrier density $\left(\sim \pm 10^{11} \mathrm{~cm}^{-2}\right)$ that greatly exceeds the density in the bulk $\left(\sim 10^{10} \mathrm{~cm}^{-2}\right) . J_{\text {ex }}$, which certainly does not have a supercurrent mediated by trivial states at the graphene-vacuum edge, demonstrates even node lifting through zero top gate voltage, when density at the edges is close to the density in the bulk [Figs. 3(e) and 3(f)]. The lack of side gate voltage sensitivity appears to rule out the contribution of the edge as the cause of the anomalous interference pattern. In the following we consider alternative mechanisms that are known to modify the magnetic interference patterns and discuss whether they could explain the observed behavior.

Geometric effects resulting in nonlocal supercurrent may yield unconventional interference patterns. For high aspect ratio junctions $(L / W \gtrsim 1)$, the supercurrent from trajectories with reflections from the vacuum edges of the junction 
(a)

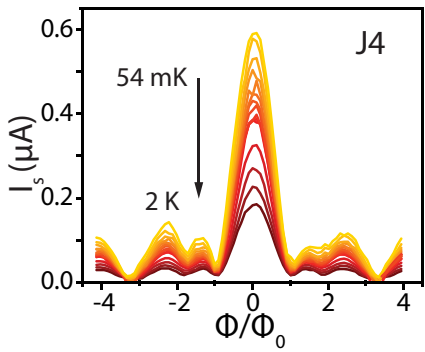

(b)

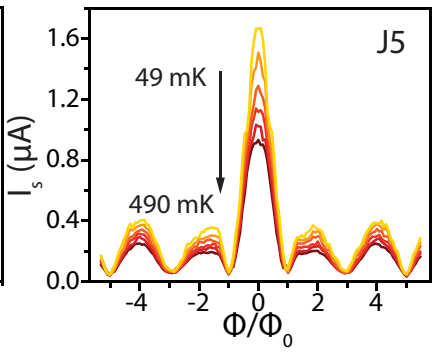

of trivial edge channels were exhaustively considered; in all cases regions of anomalous periodicity persisted. Our observations rule out likely explanations such as a nonsinusoidal CPR, large current densities at the graphene-vacuum edges, and nonlocal supercurrent contributions. While a topological state with a $4 \pi$-periodic CPR would explain our observation, no such state has been predicted to exist in graphene. Further studies of the observed interference behavior and related corroborating measurements, such as Shapiro steps, are clearly needed.

\section{ACKNOWLEDGMENTS} switching current is changing with increasing temperature, while the anomalous pattern remains qualitatively unchanged. This observation indicates that the phenomenon is not attributable to an anomalous current-phase relation, which would have been suppressed at elevated temperatures.

cannot be neglected. This nonlocal supercurrent distribution can yield a magnetic interference pattern with a $2 \Phi_{0}$ periodicity [30,32,33]. However, all of our devices have small geometric ratios $0.07<L / W<0.33$ (see Table I). Furthermore, the results from this nonlocal distribution would change the period of interference, but would not yield a pattern with a central maximum of the same width as the side maxima. Therefore, this cannot explain the pattern with even node lifting that we observe.

Any possible explanations involving a nonsinusoidal CPR can be ruled out by measurements at increased temperature, where a $2 \pi$-periodic sinusoidal relation should be recovered $[23,24]$. Such temperature dependence is presented in Fig. 4 for junctions $J_{4}$ and $J_{5}$ showing the persistence of anomalous periodicity up to $2 \mathrm{~K}$. The variety of junction lengths studied provides further evidence that this behavior is not related to CPR. Indeed, our devices range from the short $\left(L<\xi_{0}\right)$ to the long $\left(L>\xi_{0}\right)$ ballistic regime. Junctions in these opposing limits are expected to display different CPR, suggesting that the consistent behavior across all of our devices cannot originate with the CPR.

A disordered supercurrent density concentrated at several locations along the junction could explain the lifting of the nodes of the interference pattern. Indeed, the current density becomes less uniform close to charge neutrality. However, it is unlikely that such disorder would result in preferential lifting of the even nodes.

We are led to conclude that the anomalous pattern may arise from some intrinsic property of graphene. Indeed, carrier trajectories in graphene can be influenced by the valley degree of freedom, and by specular Andreev reflections [45]. It is not clear whether either could produce the observed lifting of the even nodes and the resulting quasi- $2 \Phi_{0}$-periodic patterns.

In summary, we have explored magnetic interference patterns throughout different density regimes for ballistic graphene Josephson junctions. At high carrier density, a regular Fraunhofer pattern is observed, indicating a uniform current distribution across the width of the junctions. Remarkably, at lower carrier densities we observe a robust lifting of even nodes in the interference patterns of all devices. Temperature dependence, different junction lengths, and control

Low-temperature electronic measurements performed by C.T.K., A.W.D., A.S., and G.F. were supported by the Office of Basic Energy Sciences, US Department of Energy, under Award No. DE-SC0002765. Lithographic fabrication and characterization of the samples performed by A.S. and M.T.W. were supported by ARO Award No. W911NF16-10122 and NSF Awards No. ECCS-1610213 and No. DMR1743907. H.L., M.H.R., and F.A. acknowledge the ARO under Award No. W911NF-16-1-0132. A.W.D. was supported by the NSF graduate research fellowship DGF1106401. K.W. and T.T. acknowledge the Elemental Strategy Initiative conducted by the MEXT, Japan, and the CREST (JPMJCR15F3), JST. S.T. and M.Y. acknowledge KAKENHI (Grants No. 38000131 and No. 17H01138). I.V.B. acknowledges CityU New Research Initiatives/Infrastructure Support from Central (APRC): 9610395, and the Hong Kong Research Grants Council (ECS) Project: 9048125. This work was performed in part at the Duke University Shared Materials Instrumentation Facility (SMIF), a member of the North Carolina Research Triangle Nanotechnology Network (RTNN), which is supported by the National Science Foundation (Grant No. ECCS1542015) as part of the National Nanotechnology Coordinated Infrastructure (NNCI).

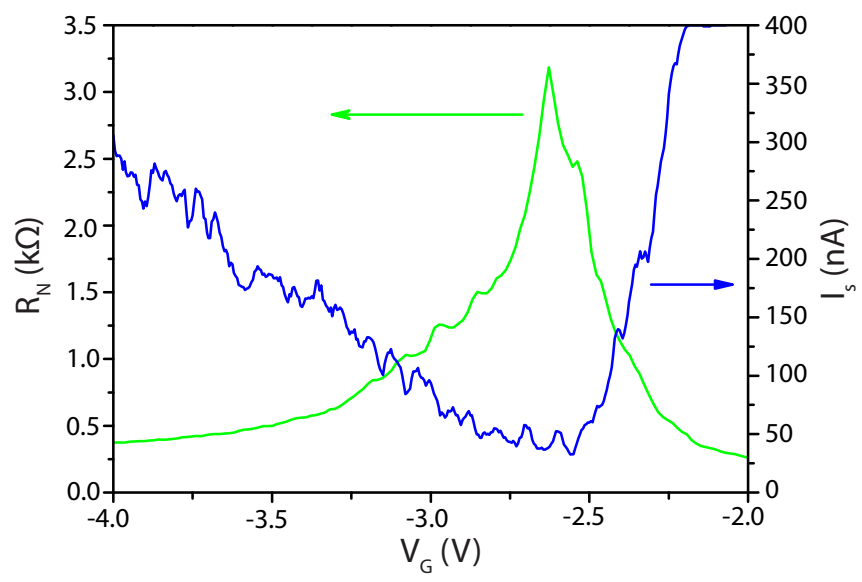

FIG. 5. The normal resistance $R_{\mathrm{N}}$ (green) and switching current $I_{\mathrm{S}}$ (blue) as a function of the gate voltage $V_{\mathrm{G}}$ for device $J_{1}$. The sharp peak of $R_{\mathrm{N}}$ indicates the DP at $V_{\mathrm{G}}=-2.65 \mathrm{~V}$. Both resistance and critical current show oscillation behavior in the Fabry-Pérot regime from $-2.65 \mathrm{~V}$ to $-3.0 \mathrm{~V}$. 


\section{APPENDIX A: DEVICES UNDER STUDY}

We fabricated Josephson junctions using exfoliated monolayer graphene crystals encapsulated in hexagonal boron nitride. The junctions' contacts are made by sputtering molybdenum rhenium (MoRe), a type-II superconductor. Further details of sample fabrication are described in our earlier work [46]. We have also junctions of different length [35]. All samples are measured in a cryogen-free dilution refrigerator with a base temperature of $35 \mathrm{mK}$. To determine the supercurrent, a bias current is continuously, linearly ramped through the junction at a repetition rate of $\sim 100 \mathrm{~Hz}$ and the voltage difference is measured across the contacts, yielding $I$ - $V$ curves. The ramp step size has a resolution of $0.3 \mathrm{nA}$, and it has been confirmed that the filtering/wiring of the device does not significantly disturb the ramp shape. The switching current $I_{\mathrm{S}}$ is then extracted as a function of back gate voltage $V_{\mathrm{G}}$ and magnetic field $B$.

\section{APPENDIX B: FABRY-PÉROT REGIME}

The normal resistance $R_{\mathrm{N}}$ and switching current $I_{\mathrm{S}}$ of $J_{1}$ are plotted in Fig. 5 as a function of gate voltage, showing a sharp resistance peak indicating the location of the Dirac point. Oscillations in both the normal resistance and switching current are visible. These oscillations result from the work function mismatch between the MoRe superconducting leads

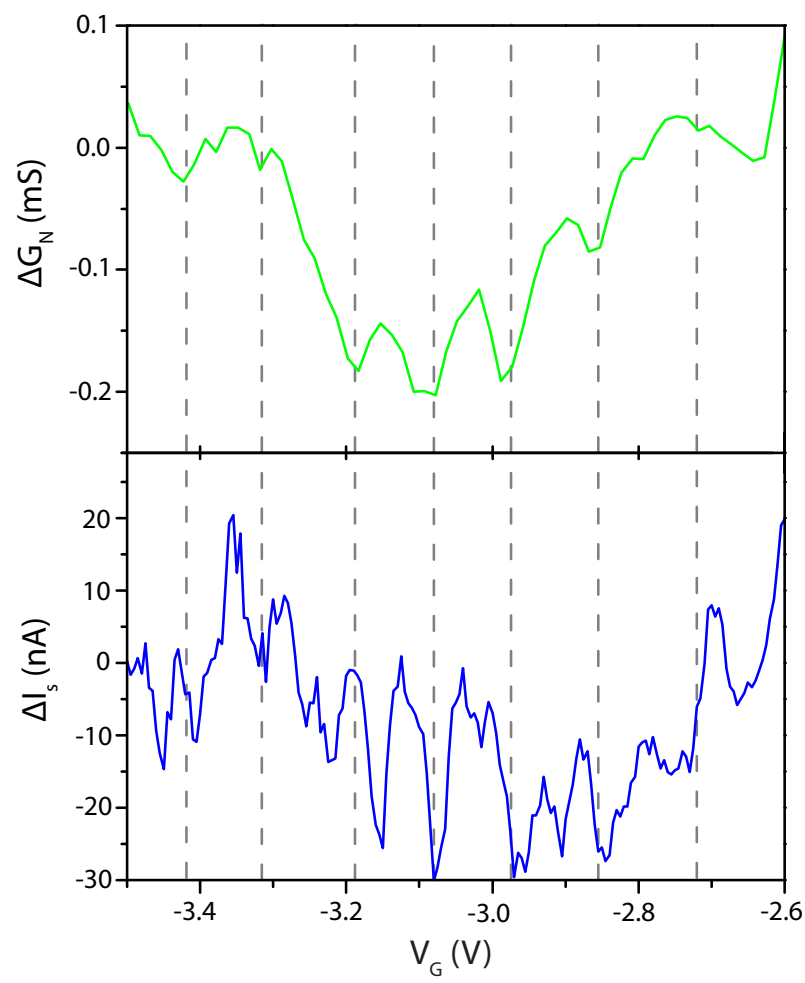

FIG. 6. Local change in normal conductance $\Delta G_{\mathrm{N}}=1 / \Delta R_{\mathrm{N}}$ and switching current $\Delta I_{\mathrm{S}}$ versus gate voltage $V_{\mathrm{G}}$ showing FabryPérot oscillations for device $J_{1} . \Delta G_{\mathrm{N}}$ and $\Delta I_{\mathrm{S}}$ were computed by subtracting a linear fit from the measured data in order to amplify the visibility of oscillations. As expected, $\Delta I_{\mathrm{S}}$ oscillates in phase with $\Delta G_{\mathrm{N}}$.

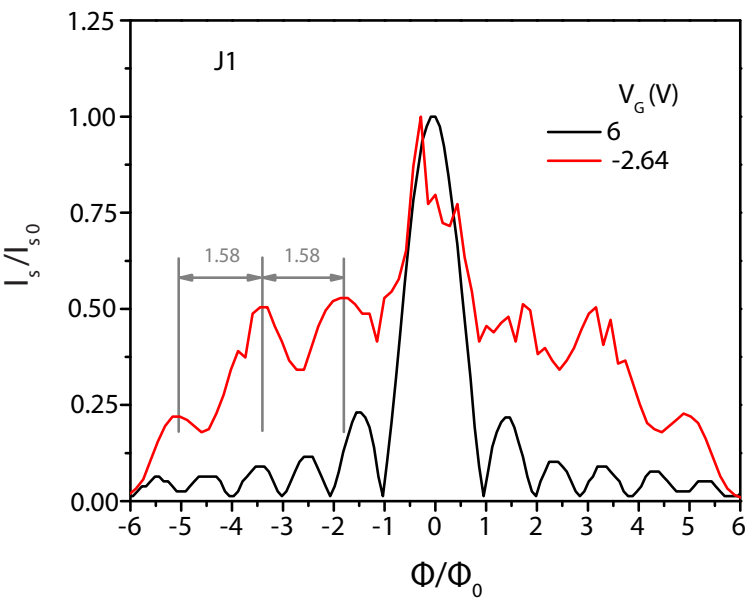

FIG. 7. The interference patterns at high-doping regime (black) and Dirac point (red). The switching-current axis is normalized by the zero-field value $I_{S 0}=I_{S}(B=0)$. While the high-doping regime demonstrates a typical Fraunhofer pattern, at the Dirac point the pattern is significantly distorted. The overall lifting of the nodes can be attributed to a disordered current density distribution. A change in periodicity is also observed by about a factor of $\sim 1.5$.

and the graphene, which yields $p n$ interfaces: MoRe locally $n$-dopes the graphene, and (in a ballistic device) a Fabry-Pérot cavity develops when the graphene bulk is $p$ doped by the back gate $V_{\mathrm{G}}$ [32]. Figure 6 plots oscillations in the normal conductance $\Delta G_{\mathrm{N}}$ and the switching current $\Delta I_{\mathrm{S}}$ versus the gate voltage $V_{\mathrm{G}}$ for device $J_{1}$, computed by subtracting a linear fit (fitted in the region displayed) from the measured $G_{\mathrm{N}}$ $\left(I_{\mathrm{S}}\right)$ (Fig. 5). This procedure isolates the oscillations, and one clearly observes regular resonances in both the supercurrent and the normal conductance. $G_{\mathrm{N}}$ and $I_{\mathrm{S}}$ oscillate in phase with each another, as expected [37]. The Fabry-Perot regime in device $J_{3}$ has been studied in detail in a previous work [35], which explored the ballisticity of junctions $J_{1-5}$.

\section{APPENDIX C: MAGNETIC INTERFERENCE PATTERN AT THE DIRAC POINT}

Figure 7 shows the magnetic interference pattern of junction $J_{1}$ taken at the Dirac point $\left(V_{\mathrm{G}} \approx-2.64 \mathrm{~V}\right)$. The dependence of critical current $I_{S}$ on $\Phi / \Phi_{0}$ is quite complex here. The critical current is never fully suppressed until after the third side lobe. This is indicative of a nonuniform current density distribution [2], which is consistent with the fact that very few conducting channels are available at the Dirac point. Conduction at the Dirac point may be affected by local impurity doping and is therefore expected to be nonuniform [47]. Note that the side lobe peak locations reveal an increase in period similar to that discussed in the main text. However, the period is not precisely doubled, but rather multiplied by $\sim 1.5$. It is possible that the same effect causing the doubling discussed in the main text is at play here as well, but complicated by the highly disordered pattern making analysis difficult. Note that due to this change in period, the pattern observed is not a simple SQUIDlike pattern as one would expect for pure edge transport [18]. 

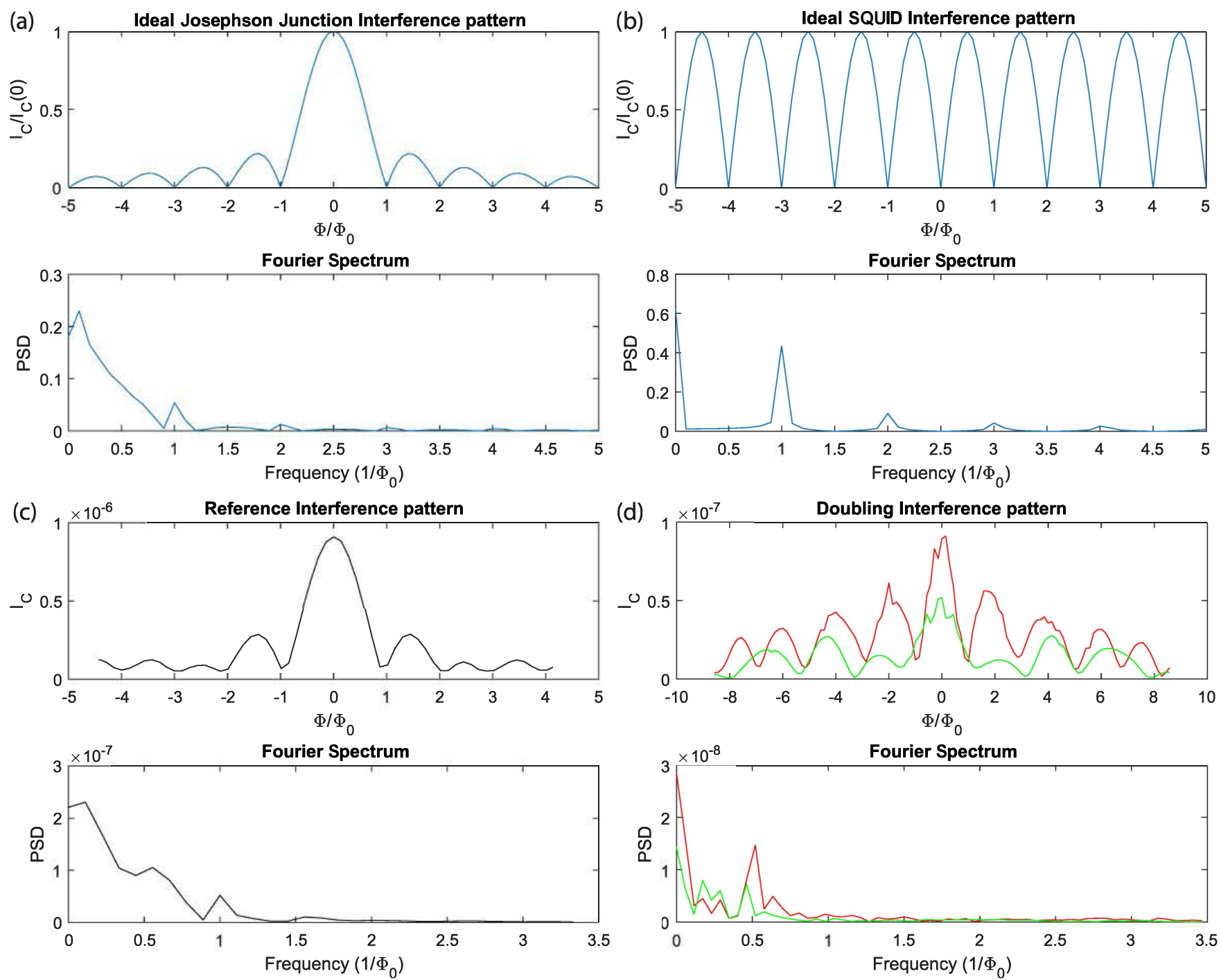

FIG. 8. Fourier analysis of magnetic interference patterns. (a) and (b): Simulated interference patterns for a uniform current distribution (standard Fraunhofer pattern) and for a SQUID (top panels) with the corresponding power spectral density (PSD) plots (bottom panels). Notably, there is a peak in the PSD corresponding to a frequency of $1 / \Phi_{0}$. (c) Interference pattern and PSD for $J_{1}$ at high electron doping $\left(V_{\mathrm{G}}=-1 \mathrm{~V}\right)$ showing the peak at $1 / \Phi_{0}$, which corresponds to the conventional $\Phi_{0}$ junction periodicity described in the text. (d) Interference pattern and PSD for $J_{1}$ in the period-doubling regime [from Fig. 1(c)]. The $1 / \Phi_{0}$ power peak is now conspicuously absent, while a peak around $1 / 2 \Phi_{0}$ is prominent.

\section{APPENDIX D: FOURIER ANALYSIS OF THE INTERFERENCE PATTERNS}

Fourier spectrum analysis of the Fraunhofer pattern is a useful tool in determining the current distribution profile [1,7]. In principle, the study of the Fourier spectrum could help us to explore the evolution of the interference pattern in the regime of period doubling. However, three factors limit the power of this analysis: (1) The frequency resolution is determined by the range of magnetic field, typically $2-3 \mathrm{mT}$ in our experiment. This range is limited by flux trapping in the superconducting leads; applying larger fields results in hysteretic distortions of the Fraunhofer pattern. (2) In order to properly Fourier-transform the Fraunhofer pattern, the phase information about the supercurrent is required. Typically, the phase is assumed to be either 0 or $\pi$, and the odd lobes are ascribed a negative sign [1]. This method is not practical when the lobes merge and the nodes between them get lifted. (3) Additional distortions from the standard Fraunhofer/SQUID patterns (particularly at low carrier densities) result in extra peaks in the frequency spectrum, especially at lower frequencies.

Nevertheless, we find that the Fourier spectrum of the Fraunhofer pattern is quite informative. In Fig. 8, we present the Fourier spectra of $I_{c}(B)$, without attempting to provide the phase information. Panels (a) and (b) provide, as a reference, standard Fraunhofer (a) and SQUID (b) interference patterns. Both show a peak corresponding to the dominant $\Phi_{0}$ periodicity of the signal. The Fraunhofer pattern shows a large low-frequency spectrum, while the SQUID exhibits stronger higher harmonics. Panel (c) shows the interference pattern measured in $J_{1}$ at high electron density $\left(V_{\mathrm{G}}=-1 \mathrm{~V}\right)$ and its frequency spectrum. Both the pattern and its spectrum are close to that of a standard Fraunhofer pattern. The spectra 


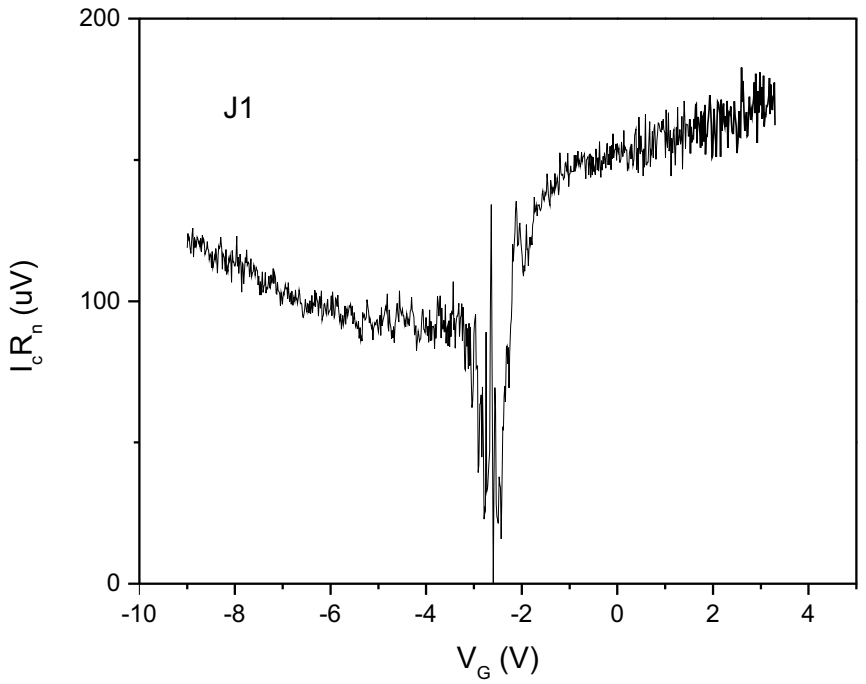

FIG. 9. The $I_{\mathrm{S}} R_{\mathrm{N}}$ product plotted versus gate voltage for device $J_{1}$.

corresponding to period doubling are shown in panel (d). A clear, strong peak corresponding to $\sim 2 \Phi_{0}$ periodicity is observed, while the $\Phi_{0}$-periodic component is practically absent (below the noise floor).

\section{APPENDIX E: CRITICAL CURRENT IN BALLISTIC JOSEPHSON JUNCTIONS}

Figure 9 shows the product of $I_{\mathrm{S}} R_{\mathrm{N}}$ plotted versus gate voltage $V_{\mathrm{G}}$ for device $J_{1}$. Note that $I_{\mathrm{S}} R_{\mathrm{N}}$ is only expected to be $\propto \Delta / e$ in short junctions. In long ballistic junctions,

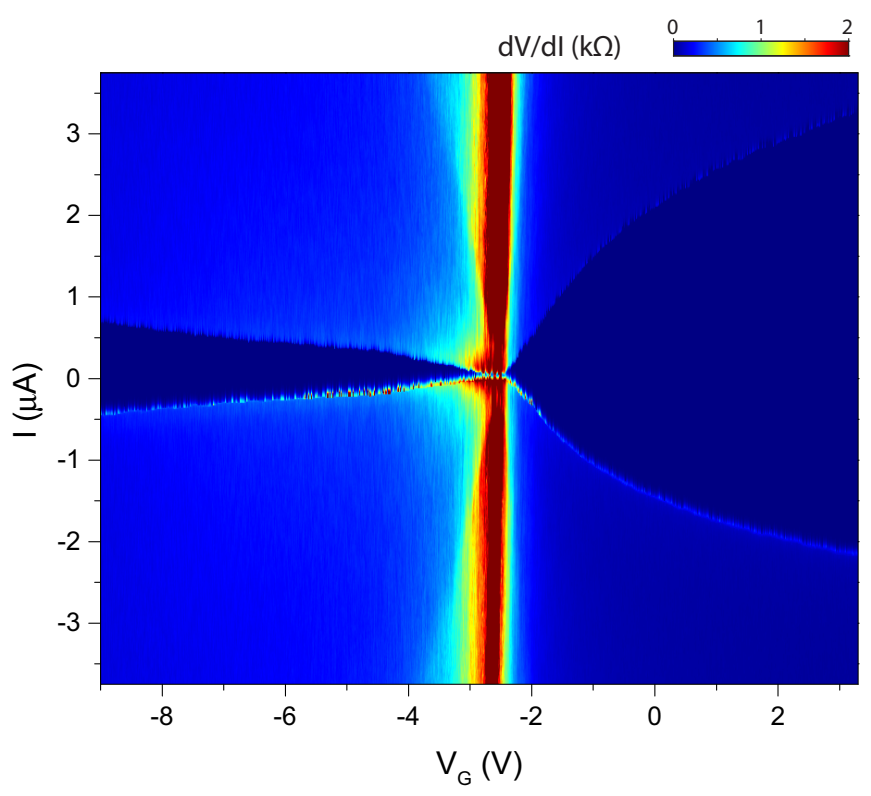

FIG. 10. Data set from Fig. 1(a) of main text extended to higher gate voltages. Bias current is swept from negative to positive; therefore the transition at negative bias corresponds to the retrapping current, while the transition at positive bias corresponds to the switching current. Retrapping current is clearly smaller than switching current, resulting in hysteresis.
$I_{\mathrm{S}} R_{\mathrm{N}} \propto v_{\mathrm{F}} / L[35,42-44]$, where $L$ is the length of the junction and $v_{\mathrm{F}}$ is the Fermi velocity, which are expected to be gate independent in graphene. A slight increase of $I_{\mathrm{S}} R_{\mathrm{N}}$ with gate voltage is explained by the imperfect contact transparency [35], which increases away from the Dirac point.

Graphene Josephson junctions typically demonstrate hysteresis between the switching and the retrapping currents, either due to self-heating or underdamped dynamics $[3,5,6]$. (The retrapping current is the current at which the junction switches from the normal to the superconducting state.) Such hysteresis is observed in all of the samples studied in this work. Figure 10 presents the same data as in Fig. 1(a) but extended to higher gate voltages, which clearly shows hysteresis.

\section{APPENDIX F: MAGNETIC INTERFERENCE FOR OTHER JUNCTIONS}

Here we show the magnetic interference patterns for devices $J_{2}$ and $J_{3}$. Following the format of Fig. 2 in the main

(a)
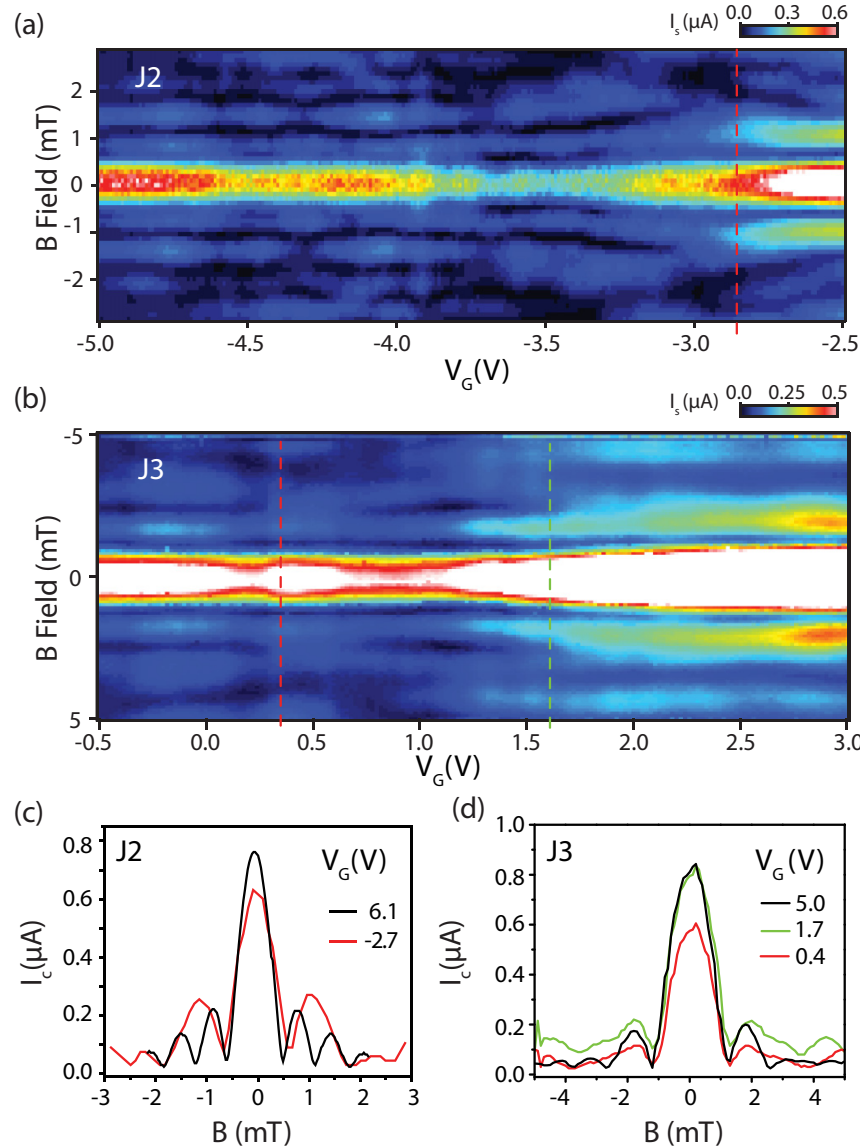

FIG. 11. Interference patterns with periodicity doubling. (a) and (b): Magnetic interference measurements at low doping for $J_{2}$ and $J_{3}$. Green and red lines mark cuts displayed in lower panels. (c) and (d): Line cuts showing supercurrent $I_{\mathrm{S}}$ as a function of quantized magnetic field $B$ for junctions $J_{2}$ and $J_{3}$, respectively. At high electron doping, a regular oscillation period is observed (black line). However, for certain $V_{\mathrm{G}}$ near the Dirac point (red, green) we find a robust lifting of the second node, resulting in an effective $2 \Phi_{0}$ periodicity. 
text, Fig. 11 shows the interference patterns for $J_{2}$ [panels (a) and (c)] and $J_{3}$ [panels (b) and (d)]. The gate maps focus on the regions around the Dirac point, and the red and green lines indicate gate voltage $V_{\mathrm{G}}$ regions of periodicity doubling. While not as clean as the devices shown in the main text, $J_{2}$ and $J_{3}$ also show clear lifting of even nodes.
[1] R. C. Dynes and T. A. Fulton, Supercurrent density distribution in Josephson junctions, Phys. Rev. B 3, 3015 (1971).

[2] M. Tinkham, Introduction to Superconductivity (McGraw-Hill, New York, 1996).

[3] H. B. Heersche, P. Jarillo-Herrero, J. B. Oostinga, L. M. K. Vandersypen, and A. F. Morpurgo, Bipolar supercurrent in graphene, Nature (London) 446, 56 (2007).

[4] F. Miao, S. Wijeratne, Y. Zhang, U. C. Coskun, W. Bao, and C. N. Lau, Phase-coherent transport in graphene quantum billiards, Science 317, 1530 (2007).

[5] X. Du, I. Skachko, and E. Y. Andrei, Josephson current and multiple Andreev reflections in graphene SNS junctions, Phys. Rev. B. 77, 184507 (2008).

[6] F. Miao, W. Bao, H. Zhang, and C. N. Lau, Premature switching in graphene Josephson transistors, Solid State Commun. 149, 1046 (2009).

[7] S. Hart, H. Ren, T. Wagner, P. Leubner, M. Mühlbauer, C. Brüne, H. Buhmann, L. W. Molenkamp, and A. Yacoby, Induced superconductivity in the quantum spin Hall edge, Nat. Phys. 10, 638 (2014).

[8] L. Fu and C. L. Kane, Josephson current and noise at a superconductor/quantum-spin-Hall-insulator/superconductor junction, Phys. Rev. B 79, 161408(R) (2009).

[9] J. Alicea, New directions in the pursuit of Majorana fermions in solid state systems, Rep. Prog. Phys. 75, 076501 (2012).

[10] F. Zhang and C. L. Kane, Anomalous topological pumps and fractional Josephson effects, Phys. Rev. B 90, 020501(R) (2014).

[11] L. Fu and C. L. Kane, Superconducting Proximity Effect and Majorana Fermions at the Surface of a Topological Insulator, Phys. Rev. Lett. 100, 096407 (2008).

[12] M. Houzet, J. S. Meyer, D. M. Badiane, and L. I. Glazman, Dynamics of Majorana States in a Topological Josephson Junction, Phys. Rev. Lett. 111, 046401 (2013).

[13] S. P. Lee, K. Michaeli, J. Alicea, and A. Yacoby, Revealing Topological Superconductivity in Extended Quantum Spin Hall Josephson Junctions, Phys. Rev. Lett. 113, 197001 (2014).

[14] V. S. Pribiag, A. J. A. Beukman, F. Qu, M. C. Cassidy, C. Charpentier, W. Wegscheider, and L. Kouwenhoven, Edgemode superconductivity in a two-dimensional topological insulator, Nat. Nanotechnol. 10, 593 (2015).

[15] L. A. Jauregui, M. T. Pettes, L. P. Rokhinson, L. Shi, and Y. P. Chen, Magnetic field induced helical mode and topological transitions in a topological insulator nanoribbon, Nat. Nanotechnol. 11, 345 (2016).

[16] E. Bocquillon, R. S. Deacon, J. Wiedenmann, P. Leubner, T. M. Klapwijk, C. Brüne, K. Ishibashi, H. Buhmann, and L. W. Molenkamp, Gapless Andreev bound states in the quantum spin Hall insulator HgTe, Nat. Nanotechnol. 12, 137 (2016).

[17] M. T. Allen, O. Shtanko, I. C. Fulga, A. R. Akhmerov, K. Watanabe, T. Taniguchi, P. Jarillo-Herrero, L. S. Levitov, and
A. Yacoby, Spatially resolved edge currents and guided-wave electronic states in graphene, Nat. Phys. 12, 128 (2015).

[18] M. T. Allen, O. Shtanko, I. C. Fulga, J. I.-J. Wang, D. Nurgaliev, K. Watanabe, T. Taniguchi, A. R. Akhmerov, P. Jarillo-Herrero, L. S. Levitov, and A. Yacoby, Observation of electron coherence and Fabry-Perot standing waves at a graphene edge, Nano Lett. 17, 7380 (2017).

[19] M. J. Zhu, A. V. Kretinin, M. D. Thompson, D. A. Bandurin, S. Hu, G. L. Yu, J. Birkbeck, A. Mishchenko, I. J. Vera-Marun, K. Watanabe, T. Taniguchi, M. Polini, J. R. Prance, K. S. Novoselov, A. K. Geim, and M. Ben Shalom, Edge currents shunt the insulating bulk in gapped graphene, Nat. Commun. 8 , 14552 (2017).

[20] F. K. de Vries, T. Timmerman, V. P. Ostroukh, J. Van Veen, A. J. A. Beukman, F. Qu, M. Wimmer, B. M. Nguyen, A. A. Kiselev, W. Yi, M. Sokolich, M. J. Manfra, C. M. Marcus, and L. P. Kouwenhoven, $h / e$ Superconducting Quantum Interference through Trivial Edge States in InAs, Phys. Rev. Lett. 120, 047702 (2018).

[21] C. Kurter, A. D. K. Finck, Y. S. Hor, and D. J. Van Harlingen, Evidence for an anomalous current-phase relation in topological insulator Josephson junctions, Nat. Commun. 6, 7130 (2015).

[22] I. Sochnikov, L. Maier, C. A. Watson, J. R. Kirtley, C. Gould, G. Tkachov, E. M. Hankiewicz, C. Brune, H. Buhmann, L. W. Molenkamp, and K. A. Moler, Nonsinusoidal Current-Phase Relationship in Josephson Junctions from the 3D Topological Insulator HgTe, Phys. Rev. Lett. 114, 066801 (2015).

[23] C. D. English, D. R. Hamilton, C. Chialvo, I. C. Moraru, N. Mason, and D. J. Van Harlingen, Observation of nonsinusoidal current-phase relation in graphene Josephson junctions, Phys. Rev. B 94, 115435 (2016).

[24] G. Nanda, J. L. Aguilera-Servin, P. Rakyta, A. Kormányos, R. Kleiner, D. Koelle, K. Watanabe, T. Taniguchi, L. M. K. Vandersypen, and S. Goswami, Current-phase relation of ballistic graphene Josephson junctions, Nano Lett. 17, 3396 (2017).

[25] A. Rasmussen, J. Danon, H. Suominen, F. Nichele, M. Kjaergaard, and K. Flensberg, Effects of spin-orbit coupling and spatial symmetries on the Josephson current in SNS junctions, Phys. Rev. B 93, 155406 (2016).

[26] H. J. Suominen, J. Danon, M. Kjaergaard, K. Flensberg, J. Shabani, C. J. Palmstrøm, F. Nichele, and C. M. Marcus, Anomalous Fraunhofer interference in epitaxial superconductor-semiconductor Josephson junctions, Phys. Rev. B 95, 035307 (2017).

[27] K. Zuo, V. Mourik, D. B. Szombati, B. Nijholt, D. J. van Woerkom, A. Geresdi, J. Chen, V. P. Ostroukh, A. R. Akhmerov, S. R. Plissard, D. Car, E. P. A. M. Bakkers, D. I. Pikulin, L. P. Kouwenhoven, and S. M. Frolov, Supercurrent Interference in Few-Mode Nanowire Josephson Junctions, Phys. Rev. Lett. 119, 187704 (2017).

[28] J. P. Heida, B. J. van Wees, T. M. Klapwijk, and G. Borghs, Nonlocal supercurrent in mesoscopic Josephson junctions, Phys. Rev. B 57, R5618 (1998). 
[29] U. Ledermann, A. L. Fauche 're, and G. Blatter, Nonlocality in mesoscopic Josephson junctions with strip geometry, Phys. Rev. B 59, R9027(R) (1999).

[30] V. Barzykin and A. M. Zagoskin, Coherent transport and nonlocality in mesoscopic SNS junctions: Anomalous magnetic interference patterns, Superlattices Microstruct. 25, 797 (1999).

[31] M. Kim, D. Jeong, G.-H. Lee, Y.-S. Shin, H.-W. Lee, and H.-J. Lee, Tuning locality of pair coherence in graphene-based Andreev interferometers, Sci. Rep. 5, 8715 (2015).

[32] V. E. Calado, S. Goswami, G. Nanda, M. Diez, A. R. Akhmerov, K. Watanabe, T. Taniguchi, T. M. Klapwijk, and L. M. K. Vandersypen, Ballistic Josephson junctions in edge-contacted graphene, Nat. Nanotechnol. 10, 761 (2015).

[33] H. Meier, V. I. Fal'ko, and L. I. Glazman, Edge effects in the magnetic interference pattern of a ballistic SNS junction, Phys. Rev. B 93, 184506 (2016).

[34] P. Rakyta, A. Kormányos, and J. Cserti, Magnetic field oscillations of the critical current in long ballistic graphene Josephson junctions, Phys. Rev. B 93, 224510 (2016).

[35] I. V. Borzenets, F. Amet, C. T. Ke, A. W. Draelos, M. T. Wei, A. Seredinski, K. Watanabe, T. Taniguchi, Y. Bomze, M. Yamamoto, S. Tarucha, and G. Finkelstein, Ballistic Graphene Josephson Junctions from the Short to the Long Junction Regimes, Phys. Rev. Lett. 117, 237002 (2016).

[36] I. V. Borzenets, U. C. Coskun, H. Mebrahtu, and G. Finkelstein, $\mathrm{Pb}$-graphene-Pb Josephson junctions: Characterization in magnetic field, IEEE Trans. Appl. Supercond. 22, 1800104 (2012).

[37] M. Ben Shalom, M. J. Zhu, V. I. Fal'ko, A. Mishchenko, A. V. Kretinin, K. S. Novoselov, C. R. Woods, K. Watanabe, T. Taniguchi, A. K. Geim, and J. R. Prance, Quantum oscillations of the critical current and high-field superconducting proximity in ballistic graphene, Nat. Phys. 12, 318 (2016).

[38] P. G. Silvestrov and K. B. Efetov, Charge accumulation at the boundaries of a graphene strip induced by a gate voltage: Electrostatic approach, Phys. Rev. B 77, 155436 (2008).

[39] I. J. Vera-Marun, P. J. Zomer, A. Veligura, M. H. D. Guimarães, L. Visser, N. Tombros, H. J. van Elferen, U. Zeitler, and B. J. van Wees, Quantum Hall transport as a probe of capacitance profile at graphene edges, Appl. Phys. Lett. 102, 013106 (2013).

[40] Y. T. Cui, B. Wen, E. Y. Ma, G. Diankov, Z. Han, F. Amet, T. Taniguchi, K. Watanabe, D. Goldhaber-Gordon, C. R. Dean, and Z. X. Shen, Unconventional Correlation between Quantum Hall Transport Quantization and Bulk State Filling in Gated Graphene Devices, Phys. Rev. Lett. 117, 186601 (2016).

[41] A. Seredinski, A. W. Draelos, E. G. Arnault, M. T. Wei, H. Li, K. Watanabe, T. Taniguchi, F. Amet, and G. Finkelstein, Quantum Hall-based superconducting interference device, Sci. Adv. 5, eaaw8693 (2019).

[42] I. O. Kulik, Macroscopic quantization and the proximity effect in S-N-S junctions, ZhETF 57, 1745 (1970) [JETP 30, 944 (1970)].

[43] J. Bardeen and J. L. Johnson, Josephson current flow in pure superconducting-normal-superconducting junctions, Phys. Rev. B 5, 72 (1972).

[44] A. V. Svidzinskii, Spatially Inhomogeneous Problems of Theory of Superconductivity (McGraw-Hill, New York, 1996).

[45] C. W. J. Beenakker, Specular Andreev Reflection in Graphene, Phys. Rev. Lett. 97, 067007 (2006).

[46] F. Amet, C. T. Ke, I. V. Borzenets, Y.-M. Wang, K. Watanabe, T. Taniguchi, R. S. Deacon, M. Yamamoto, Y. Bomze, S. Tarucha, and G. Finkelstein, Supercurrent in the quantum Hall regime, Science 352, 966 (2016).

[47] J. Martin, N. Akerman, G. Ulbricht, T. Lohmann, J. H. Smet, K. von Klitzing and A. Yacoby, Observation of electron-hole puddles in graphene using a scanning single-electron transistor, Nat. Phys. 4, 144 (2008). 